\title{
A Narrative Synthesis of the Published Literature on Antenatal Care in Low and Middle Income Countries
}

\author{
Shrestha SK ${ }^{1}$, Travaglia J², Joshi $\mathrm{C}^{3}$ \\ ${ }^{1}$ Shanta Kumar Shrestha, Maharajgunj Medical Campus, Institute of Medicine, Kathmandu, Nepal \\ 2 Joanne Travaglia, School of Public Health and Community Medicine, University of New South Wales, Sydney, Australia
}

\begin{abstract}
Background: Good quality antenatal care is one of the recommended strategies to reduce maternal and neonatal mortality and improve health outcomes of the mother and her child. The utilization and quality of antenatal care in low and middle income countries is not well documented and hence deserves further study. This is a literature review of antenatal care in low and middle income countries on its utilization, the barriers and facilitators, and the available evidence of effectiveness and quality of antenatal care.
\end{abstract}

Method: A systematic review of the published literature was conducted which also included published systematic reviews. Studies published between 2002 and 2012 were identified by searching Medline, EMBASE and CINAHL. This was supplemented by papers provided by international advisors from grey literature and snowballing search of reference list of all included papers. Only English language studies in low and middle income countries on antenatal care were included.

Results: Twenty-three papers met the inclusion criteria. Health-facility based studies reported near universal access to antenatal care, which was not the case for community based studies. Women received at least one antenatal visit but fewer women had the recommended number of visits. Socioeconomic status and women's education were the most frequent and the strongest predictors of antenatal care use. Antenatal care promoted the use of skilled birth attendant at delivery, but did not contribute to reductions in maternal mortality and stillbirths. The quality of care was reported as unsatisfactory; major issues being poor client-provider relationship and inferior quality of counselling.

Conclusion: In order to increase the utilization of antenatal care, in the short term, less educated women from socioeconomically disadvantaged households require targeting. Long-term improvements require a focus on improving female education. Further research is needed to explore the quality of antenatal care in order to validate its effect on maternal and child health.

Keywords: prenatal care; antenatal care; prepartum care; developing countries; quality of health care.

Submitted: 15 August 2014; Revised: 16 October 2014; Accepted: 20 October 2014

\section{Introduction}

Worldwide, complications during pregnancy, childbirth and postnatal period are the leading causes of death and disability among women of reproductive age (1). In 2010 , there were around 287,000 maternal deaths globally (2). A large majority of these deaths are preventable (3). Additionally, for every maternal death, an estimated 20 women suffer injury, infection or other morbidity (4). Obstetric complications such as primary haemorrhage, sepsis, complications from abortions, preeclampsia and eclampsia, and prolonged obstructed labour account for $80 \%$ of maternal deaths whilst pre-existing medical conditions aggravated during pregnancy contributes to the remaining $20 \%$ of deaths (3).

Antenatal services have been shown to have an impact on maternal and child health, especially in developing countries (5-10). The reduction of maternal morbidity and mortality, low-weight births ( $<2,500$ grams) and perinatal mortality has been shown to be related to antenatal care
(ANC) from a Skilled Birth Attendant (SBA) who is a doctor, nurse or midwife $(3,11,12)$. ANC serves as a strategy for utilization of SBAs during delivery and postnatal care $(9,13-15)$. Early ANC entry and adherence to ANC visit schedules are associated with favourable neonatal health like higher birth weights and lower infant mortality rates $(6,10,15-17)$. ANC visits provide an excellent opportunity to deliver education regarding the danger signs and symptoms during pregnancy, delivery and the postpartum period and to focus on birth spacing and family planning (18).

Provision of adequate ANC directly relates to achieving the Millennium Development Goal (MDG) 4 (reducing

\section{Correspondence:}

${ }^{3}$ Chandni Joshi (MPH, MHM, MIPH)

Centre for Primary Health Care and Equity, University of New South Wales, Sydney, Australia.

Email: c.joshi@unsw.edu.au 
child mortality rates by two-thirds) and MDG 5 (reducing maternal mortality ratio by three-quarters) by the year 2015 (19). Yet the utilisation and quality of ANC in low and middle income countries (LMICs) is not well documented and hence deserves further study (20). The study focussed on ANC use, factors associated with ANC use and the quality of care.

\section{Method}

A narrative systematic review was conducted. Medline, EMBASE and CINAHL searches were done in July 2012 for articles that included 'antenatal care', 'low and middle income countries', 'quality/utilization' and 'pregnancy outcome' and their associated terms in the title, keywords or abstract (Appendix 1). In Medline, the following search terms were used in the titles, abstracts and keywords: ('Perinatal Care' OR 'Prenatal Care' OR 'Pregnancy') AND ('Developing Countries' OR 'Low and Middle Income Countries') AND ('Health Services Accessibility' OR 'Utilization Review' OR 'Health Care Quality, Access and Evaluation' OR 'Delivery of Health Care' OR 'Quality of Health Care') AND ('Maternal Mortality' OR 'Perinatal Mortality' OR 'Mortality, Premature' OR 'Infant Mortality' OR 'Fetal Death' OR 'Fetal Mortality' OR 'Pregnancy Complications' OR 'Pregnancy Outcome'). The 'explode' option was used to increase the depth of the search. Additional papers were identified through snowballing search of reference list of all included articles and contacting interna- tional advisors for grey literature. The PRISMA checklist was followed for reporting (21).

The review was limited to articles published between 2002 and 2012 to capture the most recent literatures on this topic. The selected studies had to be published in English, in LMICs and answer at least one of the four research questions: 1) What is the utilization of ANC in LMICs? 2) What are the barriers and facilitators to ANC in these countries? 3) What is the available evidence of effectiveness of ANC in these countries? 4) What is the quality of ANC provided in these countries?

The papers were screened initially by CJ and a $20 \%$ random sample of excluded studies was reviewed and checked by JT who agreed with the exclusion of all the studies provided. Consensus was gained for uncertain articles through discussion between JT and CJ. Included studies were screened based on the title and abstract and the full paper verified through assessment of its contents based on the inclusion criteria. Data extraction was carried out by CJ and a $20 \%$ sample was verified by JT. The extracted data were entered in an MS-Excel spreadsheet which collected information on the country of study, study aim, design and methodology, characteristics of the participants, and the findings which included access, utilization, quality, effectiveness, barriers and facilitators.

Figure 1: Flow diagram of the process of identification and inclusion of articles for the narrative synthesis

일

흘

3 records included in

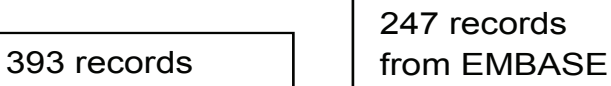

247 records from EMBASE
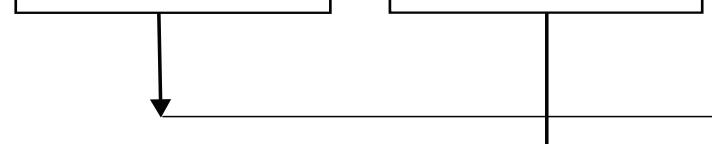

64 records from CINAHL

\section{7 records} identified from grey literature

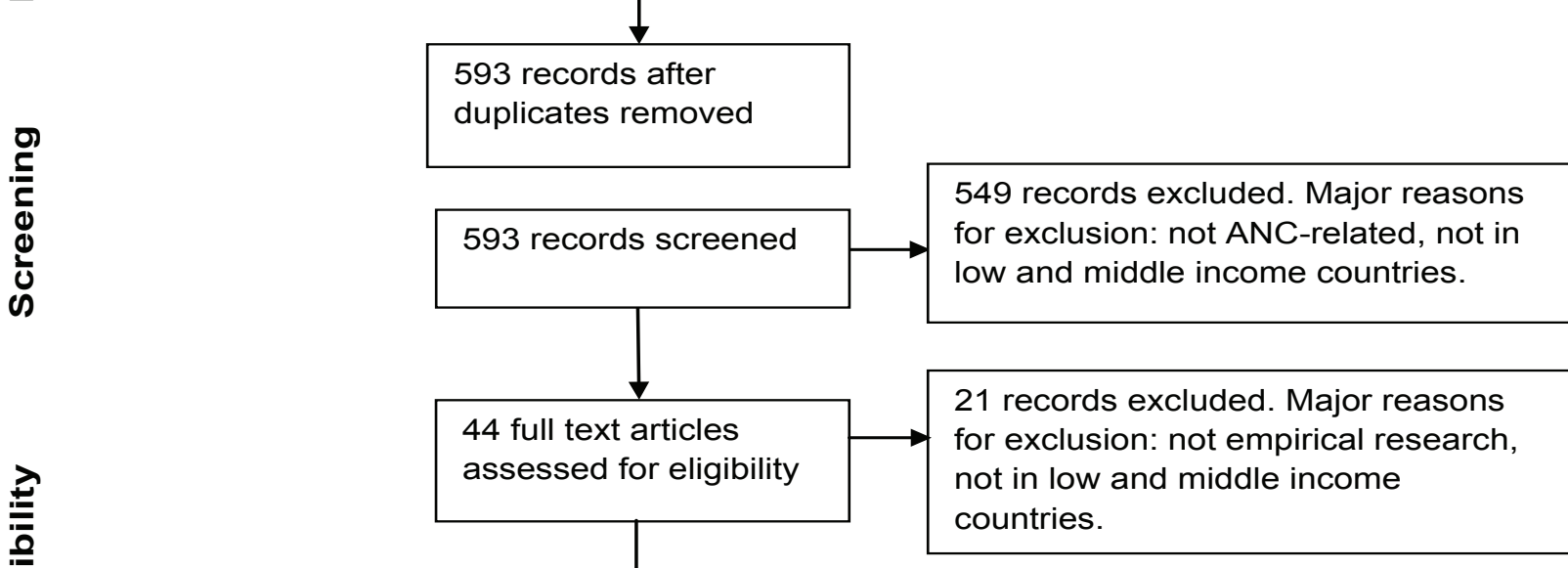
narrative synthesis 
A narrative synthesis was conducted because the studies were methodologically very diverse to combine in a meta-analysis and we wanted to fully interpret the collected evidence (22).

\section{Results}

The database search and grey literature produced 721 papers which were assessed for inclusion in the review. After the screening and verification stages, data were extracted from 23 studies. The major reasons for exclusion were that the texts were not empirical research or not ANC-related. Figure 1 shows a PRISMA flowchart of the selection process. These studies were then analysed according to the research question/s they addressed.

\section{Utilization of ANC}

Out of the 23 selected studies, twelve reported on ANC use $(7,13,17,23-31)$. Reported patterns of utilisation of ANC varied both within and across countries and service types. Out of the 12 articles, three were health facilitybased $(13,17,30)$, whereas the remaining nine were community-based $(7,23-29,31)$. Seven focussed on use of at least one ANC visit (7, 13, 24, 25, 27, 29, 30) whereas five studies reported on adequate utilization of ANC $(17,23,26,28,31)$. The number of visits considered as adequate varied among countries: three or more visits was defined as adequate for the study in Vietnam; four or more visits was considered adequate for studies in Nepal, Cambodia and Ghana while more than four visits was considered adequate in Indonesia. The extent of ANC use depended on where the study was conducted.

\section{Health-facility based studies}

In Cambodia, the majority of the women (88\%) had at least one ANC visit.(13) Majority of women in Nepal (87\%) attended more than four antenatal visits, with $94 \%$ attending at least once (17). In Gambia, a high proportion of women (99\%) attended ANC; the mean number of ANC visits reported being 3.5 (30).

\section{Non-health facility based studies}

Reported rates in India varied. In one study, $62 \%$ of women accessed ANC at least once (7). Another study in rural India showed that around $40 \%$ women had one or more ANC during their last pregnancy (29). In Taiwan $60 \%$ of women accessed ANC at least once (25). In West Sumatra, Indonesia $78 \%$ of women received more than four ANC visits (23). In Vietnam, $87 \%$ of the women received ANC from skilled personnel at least once during their last completed pregnancy (27). Another study in Vietnam comparing ANC between rural and urban areas showed that $77 \%$ women in the rural areas and $97 \%$ women in the urban areas had at least three ANC visits (31). In Nigeria, $60 \%$ of women accessed ANC at least once (24).

Two studies reported ANC use in a number of countries. A study contrasting maternal health intervention coverage (including ANC) and neonatal mortality in eight developing countries depicted the ANC coverage for Bangladesh as $25 \%$, Benin as $62 \%$, Cambodia as $29 \%$, Eritrea as $61 \%$, Haiti as $73 \%$, Malawi as $56 \%$, Nepal as $41 \%$ and Nicaragua as $82 \%$ (26). Another study on developing countries showed that receipt of at least one ANC visit was around $50 \%$ in countries like Mali, Bangladesh, Ghana and India; the only exception being Nepal where it was $15 \%$ (28).

In summary, facility-based studies report near universal ANC attendance among the study population whereas for the other studies that were community-based, there was a large variation in ANC attendance. Many women in LMICs received at least one ANC visit. However, relatively few studies reported on receipt of adequate number of ANC visits.

\section{Barriers and Facilitators of ANC}

Factors affecting the utilisation of ANC were divided into six major themes: socio-demographic factors, socio-economic factors, women's knowledge and exposure, their individual history and experiences, institutional factors and culture and tradition (see Table 1).

\section{Socio-demographic factors}

Socio-demographic factors include women's education, parity, place of residence, age, caste, ethnicity, religion, marital status, family size and structure and education levels of their husbands.

Women's education: A number of studies reported women's education as a significant factor influencing use of ANC (Table 1). The probability of women attending ANC increased with the increasing levels of their education $(7,13,24,29,31,32)$. However, one study showed that women's education was not a significant predictor of ANC utilization after controlling for other factors (23). The chances of receiving the recommended number of ANC also increased with higher levels of women's education (17). Lower levels of women's education increased the risk of late attendance at ANC (33). Women's increasing levels of education highly increased the chances of receiving ANC from a trained provider (34). The women with post-secondary education were more likely to receive the entire ANC content compared to those having no education (19).

Number of children: With the increase in the number of children that women had, the probability of receiving adequate ANC visits decreased $(6,17,23)$. Having more than one child was a risk factor for low ANC attendance $(7,13,29,33)$. One study, however, showed no statistically significant association of number of children with ANC use (24).

Place of residence: Rural women were less likely to use ANC compared to urban women $(7,24,33)$. There was a significant association between rural residence and decreased chances of using adequate ANC compared to urban residence, both regarding quantity (number and timing of visits) and content (31). Women in the urban areas were more likely to use the entire contents of ANC (19).

Age of women: Age was a significant factor in access to services, with two studies showing that young age $(<25$ years old) decreased the chances of getting adequate ANC (6, 31). Age at marriage was identified as a contributing factor; women who got married at 19 years or 
later were more likely to get ANC from a health facility than those who got married at an earlier age (29). Jat et al found that women younger than 35 years were more likely to receive ANC than those 35 years and older (7). In another study, women in the middle child-bearing ages were more likely to use ANC compared to their counterparts in the early or late child-bearing ages (24). A similar finding was shown by Tuladhar and Dhakal where women in the age group 20-29 years were more likely to have adequate number of ANC visits (17).

Caste/ethnicity/religion: The results were very contextual: women from ethnic majority groups were more likely to receive ANC compared to those belonging to minority groups $(17,27)$. Jat et al found that women from scheduled tribes in India (who are historically disadvantaged) and from Hindu religion were less likely to use ANC while Muslim women and those from non-scheduled tribes had higher chances of receiving ANC (7). On the other hand, two studies found that the statistical significance of the association between ethnicity and ANC use disappeared when other demographic variables were adjusted for (24, 33).

Education level of husbands: Husband's education was a facilitator for the use of ANC (32). Nonetheless, Amin et al found no significant association between the education of husbands and ANC utilization by their wives (34).

Marital status: Goldani et al found an association between unmarried status and inadequate ANC use (6).

\section{Socio-economic factors}

Socio-economic factors relate to financial issues and include the occupations of the women and their husbands, the household income and the community's economic status.

Economic status of the household and the community: With lower levels of household income, the chances of women attending ANC also decreased $(6,7,13,17$, $24,32,34)$. Women from poor communities had higher chances of inadequate ANC use $(6,31)$. High economic status was also found to correlate positively with receiving the entire ANC content and receiving specific components like blood pressure measurement, blood test and urine testing $(24,29)$. Poorer women were also more likely to attend ANC late or not attend at all (33).

Women's occupation: Women from agricultural and labour background were not as likely to use ANC as women from professional background (7). Women who were employed in a business or service industry had more chances of adequate ANC than housewives and labourers (17). Self-employment increased the risk for overall inadequate ANC use (31). Mumtaz and Salway found a negative association between ANC utilization and women's work for wages (32). Women's work status was not a strong predictor of ANC use in one study (29) and in another study, the association between occupation and ANC use disappeared after controlling for confounders (33).
Husbands' occupation: A study found no association between ANC from a trained provider and the occupation of women's husbands (34).

\section{Institutional structure and system}

Institutional structure and system refers to factors such as health insurance, cost of service and the characteristics of the health institution providing ANC.

Health Insurance and cost of service: In Taiwan, the introduction of national health insurance providing free ANC increased its utilization (25). The exemption of user charges in the public sector of Cambodia enabled high access to ANC (13). In Bolivia, nationwide social insurance for maternal and child health care increased ANC use in public facilities. Brazilian women with public insurance were less likely to have adequate ANC compared to those with private insurance (6).

Characteristics of health institution providing ANC: Characteristics of health institutions include location, service hours and demand, public or private services, and administrative requirements. Distance to the health centre and long waiting times at the centre were barriers to ANC use (32). The 24 hour availability of a health worker in the health institution of Cambodia was associated with high access to ANC (13). The chances of ANC use by women decreased with the increase in the size of the population served by a health facility in Nigeria (24).

Women in Vietnam had less chances of getting adequate ANC when they exclusively used a private health centre (31). Conversely, in Tanzania, greater number of women using private health facilities received the entire ANC content compared to those women using government health facilities (36).

Majority of women (86\%) in Ghana had at least one ANC; the main reason was that registration was necessary to avoid delays or annoyance to health workers in case of an institutional delivery. However, in this study, a very small proportion (35\%) had adequate ANC since the women considered that ANC was not very useful and was of low quality (28).

\section{Knowledge and exposure}

Media exposure: Women's exposure to radio, television, newspapers and magazines was found to be a strong predictor of increased ANC use in two studies. These women were more likely to use the entire ANC content than those having no media exposure $(19,29)$. Contrary to these findings, two studies found no consistent significant association of media exposure with ANC use $(24,34)$.

Knowledge and use of family planning: Women who used family planning were more likely to attend ANC when pregnant and were more likely to use the entire ANC content than those women who did not use family planning $(19,29)$. Those who approved the idea of using family planning were also more likely to utilize ANC compared to women who did not (24). Women who discussed family planning with their husbands had higher chances of using 
ANC than those who did not have such discussions (37).

\section{Culture and tradition}

Culture and tradition includes cultural values and gender roles. In Nepal, women's relationship with the household members, especially the mother-in-law and husband, greatly determined their access to ANC (32). Gender values and norms restricted women in Nepal from accessing information on reproductive health (32). In Gambia, women initiated ANC as late as in third trimester, mainly because they preferred to keep their pregnancy secret until it was noticed by family members $(28,30)$. However, in Indonesia, traditional beliefs were not associated with the number of ANC visits (23).

\section{Individual history and experiences}

Obstetric history: In the study by Bbaale, a higher portion of women suffering from a pregnancy-related problem in their past pregnancies used the entire ANC content compared to those who had normal pregnancies (19). Tran et al did not find any association between high risk pregnancy and ANC adequacy (31).

Autonomy: Women's increased autonomy resulted in increasing use of ANC in several studies. In Nepal, women who had control over their own earnings and/or had their say in decision-making during large household purchases had a greater chance of receiving ANC compared to their counterparts (37). Women's ability to travel alone had a positive impact on their use of ANC in Pakistan (32). Women's autonomy was not a significant factor in access to ANC in a study in India (29).

\section{Effectiveness of ANC}

Seven studies assessed the effectiveness of ANC (5-8, $13,17,38)$, most of them reporting positive effects. Use of ANC increased the likelihood of using skilled birth attendant at delivery and postnatal care (7). ANC safeguarded maternal health: decreased incidence of anaemia, pregnancy induced hypertension and preterm labour $(8,17)$ and promoted positive pregnancy outcomes, including a reduced risk of low birth weight and preterm babies (6, 17). Compared with women who had neonatal deaths, those with a live birth had received better quality ANC with body weight measurement, blood and urine tests, full course of iron tablets consumption, tetanus toxoid injections, abdominal examination and ultrasonography (5).

However, use of ANC did not significantly reduce maternal mortality (38). In addition, there was no association between the number of ANC visits and maternal complications or perinatal outcomes like birth weight and still birth (13).

Table 1: Summary of factors affecting the utilization of ANC

\begin{tabular}{ll}
\hline Factors affecting use of ANC & \multicolumn{1}{c}{ Studies $^{*}$} \\
\hline Socio-demographic factors & \\
Women's education & $7,13,17,19,23,24,29,31-34$ \\
Number of children & $6,7,13,17,23,24,29,33$ \\
Place of Residence & $7,19,24,31,33$ \\
Age of women & $6,7,17,24,29,31$ \\
Caste/ethnicity/religion & $7,17,24,27,33$ \\
Husbands' education & 32,34 \\
Marital status & 6
\end{tabular}

Socio-economic factors

Economic status of the household and the community

$6,7,13,17,19,24,29,31-35$

Women's occupation

$7,17,29,31-33$

Husbands' occupation

34

Institutional structure and system

Health insurance and cost of service

$6,13,25$

Characteristics of the health institution providing ANC

$13,17,19,24,28,31,32,36$

\section{Knowledge and exposure}

Media exposure

$19,24,29,34$

Family Planning

$19,24,29,37$

\section{Culture and tradition}

Culture and tradition

$23,28,30,32$

\section{Individual history and experiences}

Obstetric history

19, 31

Autonomy of women

$29,32,37$

* figures refer to the reference number in Reference section at the end of this paper.. 


\section{$\begin{array}{lll}\text { Citation } & \text { Study design } & \text { Study settings }\end{array}$}

Agus \& Horiuch Data from convenience sampling, Rural Indonesia (2012)

Amin et al.

(2010)

Babalola \& Fa-

tusi (2009)

Barros et al

(2012)

$\vec{v}$

Bbaale (2011)

Chen et al.

(2003)
Data from convenience sampling
questionnaires distributed among women with the help of midwives.

Survey using a structured questionnaire.

Data from 2005 National HIV/

AIDS and Reproductive Health

Survey.

Data from demographic and

health survey and multiple indicator cluster survey.

Data from Uganda Demographic and health survey.

Survey using questionnaires handed out to women.

Data from Demographic and

Health Surveys.

Quantitative study using structured interview.
Rural Bangladesh

Nigeria

health survey and

multiple indicator cluster survey.

Uganda

Taiwan

Fenn et al

(2007)

8 developing

countries: Bangla-

desh, Benin, Haiti,

Malawi, Nepal,

Nicaragua

Peri-urban India

\section{Study aims}

Study participants

To describe the factors related to low utilization of ANC services among pregnant women in a rural area in Indonesia.

To examine socioeconomic differentials in maternal and child health-seeking behavior in rural Bangladesh. and state-level factors.

54 countries having To compare how different coverage indidemographic and cators perform in terms of equity.

Cambodia, Eritrea practices and postnatal care.
To examine the determinants of maternal services utilization in Nigeria, with a focus on individual, household, community

To describe intra- and inter-household differences in antenatal care, delivery

A total of 145 of 200 married women of reproductive age who were pregnant or had experienced birth were included.

3498 currently married women from 128 remote villages of Bangladesh.

2148 women who had a baby during the five years preceding the survey.

Women who had children born in the 2 years before the Multiple Indicator Cluster Survey and children born up to 3 years before the Demographic and Health Survey.

To investigate the factors associated with the use of antenatal care content to inform policy makers of the pertinent factors that need to be influenced by policy.

This paper focussed on the determinants of antenatal care use in Taiwan and provided a comparison of access to care before and after National Health Insurance was implemented in 1995.

To determine the extent of within-country inequities in neonatal mortality and effective intervention coverage.

8531 women aged 15-49 years.

Data for this analysis was selected from two cohorts totalling approximately 5000 pregnant wome living in Taiwan. The questionnaire was handed out in all 23 administrative districts. The first cohort consisted of 1662 women, who delivered on May 15 , May 16 or May 17, 1989. The second cohort comprised 3626 women who had a birth during a 5-day study period (February 12-16, 1996).

85,406 women in reproductive age group from eig countries.

892 married women aged less than 50 years living in a peri-urban area of Kanpur city in Uttar Pradesh. 


Fujita et al. A health facility-based retrospec- Cambodia

(2005)

Jat et al. (2011) Cross sectional study

Kirkwood et al. Mixed methods: in-depth in2008) terviews with key informants, focused group discussions, observations, case studies, illness/ death narratives, social mapping, pile sorts, free listing, pictures, hypothetical scenarios, crosssectional surveys, intervention workshops, trials of

improved practices and piloting.
India

To analyze the situation of ANC attendance in the capital of Cambodia, Phnom Penh, and the effect of ANC attendance on delivery and newborn outcome, in order to establish a strategy for ANC in the national reproductive health program in Cambodia.

To explore three dimensions of women's position within their household-decision making, employment and influence over earnings, and spousal discussion of

family planning and their use of maternal health care.

To analyze utilization of antenatal care and skilled birth attendance among Vietnamese women of reproductive age in relation to social determinants with the aim to reveal health inequities and identify disadvantaged groups.

To investigate trends in prenatal care use and its association with low birth weight in Brazil.

To estimate the effects of individual, community and district level characteristics on the utilisation of maternal health services with special reference to antenata care, skilled attendance at delivery and postnatal care.

Developing countries: Bangladesh, Ghana, India, Mali and Nepal

This paper summarizes lessons learned from formative research conducted in Bangladesh, Ghana, India, Mali and Nepal to inform the development of newborn health interventions.
Women who delivered during 1 month at all the public and private health facilities in Phnom Penh.

Women aged 15-49 who had given birth in the three years preceding the survey.

1,023 women who had given birth to a live child two years preceding the survey.

All singleton live births to resident families of southeast Brazil (6,750 mother-child pairs in the 1978-79 cohort and 2,846 mother-child pairs in 1994).

15,782 ever-married women aged $15-49$ years residing in Madhyapradhesh state who delivered a child during the three years preceding the survey.

Women of reproductive age from five countries in South Asia: Bangladesh (Mirzapur and Sylhet), India (Rajasthan and Uttar Pradesh), Nepal (Makwanpur); Sub-Saharan Africa: Ghana (Brong Ahafo), Mali (six districts to give national representativeness). 
way $(2007)$

was collected through observa-

tion, interview with women, their

husbands and mothers-in-law

and focus group discussion with

women and men. The quantitative

data was a secondary data analy-

sis of the Pakistan Fertility and

Family Planning Survey 1996-97

Neupane \& Doku Nepal Demographic and Health (2012) Survey.

Pallikadavath et al. (2004) (2010) generate a list of all women who delivered between October 1 , 1997 and September 30, 1998. All of these women were invited to participate and were interviewed between six weeks and one year after delivery using a standardised questionnaire.

Nepal
Pattinson (2004) Data was collated from the 44 Perinatal Problem Identification Program sentinel sites spread throughout South Africa.

Pembe et al. A cross-sectional study was conducted in 18 primary health facilities.

The Farafenni demographic surveillance system was used to

South African countries

Tanzania

Gambia (2002)

To provide an integrated empirical analysis of quantitative and qualitative data in order to identify the ways in which the gender order in Punjab, Pakistan influenced women's access to ANC. Secondly, to use the empirical observations as a springboard for critiquing prevalent theoretical approaches to investigating gendered influences on women's reproductive health in South Asia.

This study aimed at investigating the factors determining the timing of first prenatal care visit and the number of prenatal care visits among a national representative sample of Nepali women.

To examine factors associated with use of antenatal care in rural areas of northIndia, to investigate access to specific critical components of care and differences in the pattern of services received via health facilities versus home visits.

To assess the potential for reducing the perinatal mortality related to spontaneous preterm delivery.

To assess quality of antenatal care with respect to providers' counselling of pregnancy danger signs in Rufiji district, Tanzania.

To provide information from a community-based study of 623 women who had recently given birth in the Farafenni area. Information on how, when, and why care was accessed, and what type of care and information were obtained. Women were asked about their experiences during prenatal, delivery and postpartum periods.
The qualitative study was carried out in a village in northern Pakistan with 15 women, 15 husbands and five mothers-in-law. The quantitative study collected data on 1209 Punjabi ever married women aged 15-49 years who had given birth within 3 years prior to the survey.

4,136 women aged 15-49 years who had delivery within three years prior to the survey.

90,303 ever-married women aged 15-49 who gave birth in the last 3 years preceding the survey from 26 states comprising $99 \%$ of India's population.

3,045 perinatal deaths among 78,343 births of $\geq 1000$ grams

Thirty two providers were observed providing antenatal care to 438 pregnant women. Information on counselling on pregnancy danger signs was collected by an observer. Exit interviews were conducted to 435 women.

623 women who had recently given birth in the Farafenni area. 
Table 2: Summary of studies included in the review (continued)

Nyamtema et al. Cross-sectional descriptive study Rural Tanzania (2012)

Tran et al. (2012) tured questionnaires.

Quantitative using structure ques- Vietnam tionnaire.

Tuladhar \&

Prospective descriptive study.

Nepal
To assess in a broader view the quality of antenatal care services and underlying factors in Kilombero district, Tanzania.

To identify factors, demographic, social and economic, possibly associated with three ANC indicators: number of visits, timing of visits and content of services.

Also, to compare the patterns of association of such factors between a rural and an urban context in northern Vietnam.

To identify the determinants of antenatal 322 women delivering at Nepal Medical College

care attendance and its impact on mater- Teaching Hospital. nal / perinatal outcome.
All clients $(\mathrm{N}=363)$ who came for antenatal clinics on the day of study in the 2 hospitals, 2 health centers and 7 dispensaries were included in this study. 2132 pregnant women were followed from identification of pregnancy until birth in two Health and Demographic Surveillance Sites in DodaLab and FilaBavi.

nal/ perinatal outcome.




\section{Quality of ANC}

Seven studies reported on the quality of the ANC provided $(5,17,28,30-32,39)$. The major issues were the attitudes of health care workers and the quality of counselling provided on pregnancy-related matters.

Negative attitudes of health care workers and poor clientprovider relationship were reported as major barriers to ANC attendance. Women perceived public service as having poor quality and rude and inconsiderate staffs (32). The quality of health education provided on matters like pregnancy danger signs, place to visit during emergencies, importance of institutional deliveries and of postnatal care was reported to be very poor, with very few women being informed on such matters $(28,30,39)$. ANC providers had poor counselling skills and the health education provided by them were ineffectual (28). Though the counselling was suboptimal, other components of ANC were provided to the majority of women (39). However, another study found the ANC components provided were inadequate compared to the national recommendations, and this was especially true for the private sector (31).

Women who attended an adequate number of ANC visits had better quality ANC in terms of the ANC content, compared to the women who did not have adequate ANC (17). Interestingly, nurse auxiliaries, considered as unskilled providers, were better at informing clients of danger signs compared to the registered nurses, who were considered to be skilled providers (39).

\section{Discussion}

Articles published in English in peer-reviewed journals between 2002 and 2012 were included in this review. The review reports on the extent of ANC use in low and middle income countries and the effectiveness and quality of ANC, and has identified several factors pertinent to ANC use. However, these results are to be viewed on a contextual basis and might differ from country to country.

Health-facility based studies report near universal access to ANC, which is not the case for community-based studies. Comparison on utilization of ANC should be taken into consideration; also, issues like when the study was undertaken, whether the focus of the study was on adequate ANC or any ANC contact and whether the study was conducted in rural or urban setting should be equally considered.

The most frequently reported factor influencing the use of ANC was socio-economic status. Low socioeconomic status increased women's probability of attending ANC late or not attending at all. Such women were more likely to have inadequate ANC and less likely to receive specific ANC components like blood pressure measurement and blood and urine tests. Greater affordability of health services and related costs, including transportation costs and increased access to health information, among women of higher socioeconomic status compared to women from lower socioeconomic status may explain these associations (20). Many developing countries have adopted financial incentives for women to attend ANC. This has resulted in its increased utilization by reducing their outof-pocket expenditure for health care and its associated costs (40-42).

Women's education was the second most frequently reported factor influencing ANC use. Educated women had better chances of having any contact with ANC, attending ANC at an earlier stage of pregnancy, having an adequate number of ANC visits and receiving care from a trained provider. A number of pathways have been suggested through which women's education levels might affect their health care utilization. These include possessing greater knowledge on the importance of health care, facing fewer difficulties to access health services and enhanced ability to select the most appropriate service for their needs (43). Quality of care is an equally important factor for the continuation of ANC (20). ANC has to be adequate both in quality and quantity in order to have a positive impact on maternal and child health (44). This review found the quality of ANC to be inadequate and the major issues were poor client-provider relationships and the low quality of counselling provided on pregnancy-related matters. The ANC visit provides a forum to inform the women about matters that may positively influence health outcomes for the mother and her child (45). ANC currently seems to be a missed opportunity for providing health education and essential interventions $(9,28)$. Very few studies reported on the components of the ANC and hence the adequacy of the quality of care provided is not known. The quality and timing of ANC could be associated with maternal mortality $(18,46)$.

Women in several African countries didn't view ANC as important other than a pathway for registration for institutional delivery $(47,48)$. Culture and tradition influence women's perception towards their health and the use of health services. The concept of preventive health services is alien in South Asian culture as health care services are perceived as curative only (49). Women do not seek ANC during pregnancy as they perceive ANC as a treatment for some kind of illness (48).

The review captured the latest literature on antenatal care in low and middle income countries and elaborated on its magnitude of access, quality of care and its effectiveness on the maternal and child health outcomes. However, this review did not include non-English language studies. Nonetheless, much of the research from the developing part of the world might not be published in peer-reviewed journals but may be available as grey literature in local languages. To mitigate publication bias, grey literature were collected from international advisors.

\section{Conclusion}

Women's socio-economic status and education were the most recurring and strong predictors of women's ANC use. Hence, in the short term, less educated women from socioeconomically disadvantaged households require targeting. Long-term improvements require a focus on improving female education. Few studies reported on the effectiveness of ANC and the quality of care. Further research could be helpful to explore the quality of ANC in 
order to validate the effect of ANC on maternal and child health.

\section{Appendix 1: Search terms}

1. Medline: 393 search results

ANC:

Perinatal care/or Prenatal care/or Pregnancy

\section{Low and Middle Income Countries:}

Developing countries/ or (Low and middle income countries).mp.

Accessibility:

Health services accessibility/ or "Utilization review"/ or "Health care quality, access, and evaluation"/ or "Delivery of health care"/or "Quality of health care"/

\section{Outcomes:}

Maternal mortality/ or Perinatal mortality/ or Mortality, premature/ or Infant mortality/ or Fetal death/ or Fetal mortality/ or Pregnancy Complications/ or Pregnancy Outcome/

2. EMBASE: 247 search results

ANC:

Prenatal care/ or Pregnancy/

Low and Middle Income Countries:

Developing country/ or (Low and middle income countries).mp.

Accessibility:

"Utilization review"/ or Health care utilization/ or Health care delivery/ or Health care quality/

\section{Outcomes:}

Maternal mortality/ or Maternal morbidity/ or Perinatal mortality/ or Fetus mortality/ or Infant mortality/

3. CINAHL: 64 search results

ANC:

(MH "Pregnancy") or (MH "Prenatal care")

Low and Middle Income Countries:

(MH "Developing countries") or (Low and middle income countries).mp.

Accessibility:

(MH "Health Resource Utilization") or (MH "Health Services Accessibility") or (MH "Utilization Review") or ( $\mathrm{MH}$ "Quality of Health Care")

\section{Outcomes:}

(MH "Maternal Mortality") or (MH "Perinatal Death") or (MH "Infant Mortality") or (MH "Pregnancy Complications") or MH "Pregnancy Outcomes"

\section{Abbreviations}

ANC: Antenatal Care; LMIC: Low and Middle Income Country; MDG: Millennium Development Goal.

\section{Competing interests}

The authors declare that they have no competing interests.

\section{Authors' contributions}

SKS helped design the study and analyze the data, suggested improvements for the review report and drafted the manuscript. JT supervised each process of the systematic review and revised the manuscript. CJ designed the study, identified the studies for inclusion in the systematic review, conducted the data extraction and prepared the review report. All authors read and approved the final manuscript.

\section{Acknowledgement}

We are grateful to Dr. Shanti Raman, Clinical Senior Lecturer at the University of Sydney for helping in revising the study design and identifying relevant papers to be included in the review.

\section{References}

1. World Health Organization. The world health report 2006-working together for health. Geneva: WHO, 2006.

2. World Health Organization, United Nations Children's Fund, United Nations Population Fund, World Bank. Trends in Maternal Mortality: 1990-2010. Geneva: WHO, 2012.

3. World Health Organization. The World Health Report: Make Every Mother and Child Count. Geneva: WHO, 2005.

4. Hogan MC, Foreman KJ, Naghavi M, Ahn SY, Wang M, Makela SM et al. Maternal mortality for 181 countries, 1980-2008: a systematic analysis of progress towards Millennium Development Goal 5. The Lancet. 2010;375(9726):1609-23. PMID: 20382417

5. Ghosh R, Sharma AK. Intra- and inter-household differences in antenatal care, delivery practices and postnatal care between last neonatal deaths and last surviving children in a peri-urban area of India. Journal of Biosocial Science. 2010 Jul;42(4):511-30. PMID: 20202272.

6. Goldani MZ, Barbieri MA, Silva AAM, Bettiol H. Trends in prenatal care use and low birthweight in southeast Brazil. American Journal of Public Health. 2004;94(8):1366-71. PMID: 15284045. PMCID: PMC1448457.

7. Jat TR, $\mathrm{Ng} \mathrm{N}$, San Sebastian M. Factors affecting the use of maternal health services in Madhya Pradesh state of India: a multilevel analysis. International Journal for Equity in Health. 2011;10(1):59. PMCID: PMC3283453

8. Pattinson RC. Are deaths due to prematurity avoidable in developing countries? Tropical Doctor. 2004;34(1):7-10. PMID: 14959961.

9. Stanton C, Blanc AK, Croft T, Choi Y. Skilled care at birth in the developing world: progress to date and strategies for expanding coverage. Journal of Biosocial Science. 2007;39(1):109-20. PMID: 1652222.

10. Yakoob MY, Menezes EV, Soomro T, Haws RA, Darmstadt GL, Bhutta ZA. Reducing stillbirths: behavioural and nutritional interventions before and during pregnancy. BMC Pregnancy \& Childbirth. 2009;9 (Suppl 1):S3. PMID: 19426466. PMCID: PMC2679409.

11. Adekanle DA, Isawumi Al. Late antenatal care booking and its predictors among pregnant women in south western Nigeria. Online Journal of Health and Allied Sciences. 2008;7(1):1-6.

12. Petrou S, Kupek E, Vause S, Maresh M. Antenatal visits and adverse perinatal outcomes: results from a British population-based study. European Journal of Obstetrics \& Gynecology and Reproductive Biology. 2003;106(1):40-9. PMID:12475580

13. Fujita N, Matsui M, Srey S, Po CS, Uong S, Koum K. Antenata care in the capital city of Cambodia: current situation and impact on obstetric outcome. Journal of Obstetrics and Gynaecology Research. 2005;31(2):133-9. PMID: 15771639

14. Chakraborty N, Islam MA, Chowdhury RI, Bari W. Utilisation of postnatal care in Bangladesh: evidence from a longitudinal study. Health \& Social Care in the Community. 2002;10(6):492-502. PMID:12485137.

15. Mrisho M, Obrist B, Schellenberg JA, Haws RA, Mushi AK, Mshinda $H$, et al. The use of antenatal and postnatal care: perspectives and experiences of women and health care providers in rural southern Tanzania. BMC pregnancy and childbirth. 2009;9:10. PMCID: PMC2664785

16. Cokkinides V. Health insurance coverage-enrollment and adequacy of prenatal care utilization. Journal of Health Care for the Poor and Under-served. 2001;12(4):461-73. PMID: 11688196 
17. Tuladhar H, Dhakal N. Impact of Antenatal Care on Maternal and Perinatal Outcome: A Study at Nepal Medical College Teaching Hospital. Nepal Journal of Obstetrics and Gynaecology. 2011;6(2):37-43.

18. World Health Organization. Antenatal care in developing countries. Promises, achievements and missed opportunities: An analysis of trends, levels and differentials, 1990-2001. Geneva: WHO, 2003.

19. Bbaale E. Factors influencing the utilisation of antenatal care content in Uganda. Australasian Medical Journal. 2011;4(9):516-526. PMID: 23393544. PMCID: PMC3562912.

20. Joshi C, Torvaldsen S, Hodgson R, Hayen A. Factors associated with the use and quality of antenatal care in Nepal: a population-based study using the demographic and health survey data. BMC Pregnancy and Childbirth. 2014;14:94. PMID: 24589139. PMCID: PMC3943993.

21. Moher D, Liberati A, Tetzlaff J, Altman DG. The PRISMA Group: Preferred reporting items for systematic reviews and meta-analyses: the PRISMA statement. PLoS Medicine. 2009:264:9. PMID: 20171303.

22. Centre for Reviews and Dissemination. Systematic Reviews: CRD's guidance for undertaking reviews in health care. United Kingdom: University of York, 2008.

23. Agus $Y$, Horiuchi S. Factors influencing the use of antenatal care in rural West Sumatra, Indonesia. BMC Pregnancy and Childbirth. 2012;12(1):9. PMID: 22353252. PMCID: PMC3298506

24. Babalola S, Fatusi A. Determinants of use of maternal health services in Nigeria-looking beyond individual and household factors. BMC Pregnancy and Childbirth. 2009;9(1):43. PMID: 19754941. PMCID: PMC2754433.

25. Chen CS, Liu TC, Chen LM. National Health Insurance and the antenatal care use: a case in Taiwan. Health Policy. 2003;64(1):99-112. PMID: 12644332

26. Fenn B, Kirkwood BR, Popatia Z, Bradley DJ. Inequities in neonatal survival interventions: evidence from national surveys. Archives of Disease in Childhood Fetal \& Neonatal Edition. 2007;92(5):F361-6. PMID: 17379739. PMCID: PMC2675357.

27. Goland E, Hoa DTP, Målqvist M. Inequity in maternal health care utilization in Vietnam. International Journal for Equity in Health. 2012;11(1):24. PMID: 22587740. PMCID: PMC3477052.

28. Kirkwood B, Sharmin T, Ali N, Darmstadt G, Winch P, Bazzano A, et al. Developing community-based intervention strategies to save newborn lives: lessons learned from formative research in five countries. Journal of Perinatology. 2008;28(2):S2-S8. PMID: 19057564. PMID: 19057564

29. Pallikadavath S, Foss M, Stones RW. Antenatal care: provision and inequality in rural north India. Social Science \& Medicine. 2004;59(6):1147-58. PMID: 15210087

30. Telfer ML, Rowley JT, Walraven GEL. Experiences of mothers with antenatal, delivery and postpartum care in rural Gambia. African Journal of Reproductive Health. 2002;6(1):74-83. PMID: 12476731.

31. Tran TK, Gottvall K, Nguyen HD, Ascher H, Petzold M. Factors associated with antenatal care adequacy in rural and urban contexts-results from two health and demographic surveillance sites in Vietnam. BMC Health Services Research. 2012;12(1):40. PMID: 22335834. PMCID: PMC3305637.

32. Mumtaz Z, Salway SM. Gender, pregnancy and the uptake of antenatal care services in Pakistan. Sociology of Health \& Illness. 2007;29(1):1-26. PMID: 17286703.

33. Neupane S, Doku DT. Determinants of time of start of prenatal care and number of prenatal care visits during pregnancy among Nepalese women. Journal of Community Health. 2012;37(4):865-73. PMID: 22134620 .
34. Amin R, Shah NM, Becker S. Socioeconomic factors differentiating maternal and child health-seeking behavior in rural Bangladesh A cross-sectional analysis. International Journal for Equity in Health. 2010;9(9):1-12. PMID: 20361875. PMCID: PMC2859349.

35. Barros AJ, Ronsmans C, Axelson H, Loaiza E, Bertoldi AD, França GV, et al. Equity in maternal, newborn, and child health interventions in Countdown to 2015: a retrospective review of survey data from 54 countries. The Lancet. 2012;379(9822):1225-33. PMID: 22464386.

36. Nyamtema AS, Bartsch-de Jong A, Urassa DP, Hagen JP, van Roosmalen J, Ifakara T. The quality of antenatal care in rural Tanzania: what is behind the number of visits? BMC Pregnancy and Childbirth 2012;12(1):70. PMID: 22823930. PMCID: PMC3434089.

37. Furuta M, Salway S. Women's position within the household as a determinant of maternal health care use in Nepal. International Family Planning Perspectives. 2006:17-27. PMID: 16723298.

38. Bulatao RA, Ross JA. Which health services reduce maternal mortality? Evidence from ratings of maternal health services. Tropical Medicine \& International Health. 2003;8(8):710-21. PMID: 12869092

39. Pembe AB, Carlstedt A, Urassa DP, Lindmark G, Nystrom L, Darj E. Quality of Antenatal Care in rural Tanzania: counseling on pregnancy danger signs. BMC Pregnancy and Childbirth. 2010; 10:35. PMID: 20594341. PMCID: PMC2907301.

40. Barber SL, Gertler PJ. Empowering women to obtain high quality care: evidence from an evaluation of Mexico's conditional cash transfer programme. Health Policy and Planning. 2009;24(1):18-25. PMID: 19022854. PMCID: PMC2724849.

41. Gertler PJ, Boyce S. An Experiment in Incentive-based Welfare: The impact of Progresa on health in Mexico. University of California, Berkeley. 2001:30-7. Royal Economic Society Annual Conference. 2003(8).

42. Morris SS, Flores R, Olinto P, Medina JM. Monetary incentives in primary health care and effects on use and coverage of preventive health care interventions in rural Honduras: cluster randomised trial. The Lancet. 2004;364(9450):2030-7. PMID: 15582060.

43. Titaley CR, Dibley MJ, Roberts CL. Factors associated with underutilization of antenatal care services in Indonesia: results of Indonesia Demographic and Health Survey 2002/2003 and 2007. BMC Public Health. 2010;10(1):485. PMID: 20712866. PMCID: PMC2933719.

44. Ciceklioglu M, Soyer MT, Öcek ZA. Factors associated with the utilization and content of prenatal care in a western urban district of Turkey. International Journal for Quality in Health Care. 2005;17(6):533-9. PMID: 16203753

45. Osungbade K, Oginni S, Olumide A. Content of antenatal care services in secondary health care facilities in Nigeria: implication for quality of maternal health care. International Journal for Quality in Health Care. 2008;20(5):346-51. PMID: 18621778.

46. Vanneste AM, Ronsmans C, Chakraborty J, De Francisco A. Prenatal screening in rural Bangladesh: from prediction to care. Health Policy and Planning. 2000;15(1):1-10. PMID: 10731229.

47. Burgard S. Race and pregnancy-related care in Brazil and South Africa. Social Science \& Medicine. 2004;59(6):1127-46. PMID: 15210086.

48. Say L, Raine R. A systematic review of inequalities in the use of maternal health care in developing countries: examining the scale of the problem and the importance of context. Bulletin of the World Health Organization. 2007;85(10):812-9. PMID: 18038064. PMCID: PMC2636485.

49. Stephenson R, Tsui AO. Contextual influences on reproductive health service use in Uttar Pradesh, India. Studies in Family Planning. 2002;33(4):309-20. PMID: 12561780 